\title{
Decorative Art Pattern Mining and Discovery Based on Group User Intelligence
}

\author{
Kangning Shen, Taizhou College, Nanjing Normal University, China \\ Rongrong Tu, Nanjing Normal University, China \\ Rongju Yao, Weifang University of Science and Technology, China \\ Sifeng Wang, Qufu Normal University, China \\ Ashish K. Luhach, The PNG University of Technology, Papua New Guinea
}

\begin{abstract}
With the continuous development of real estate and the increasing personalization of people, more and more homeowners are willing to search for and discover their preferred decorative art patterns via various house decoration websites or platforms. Through browsing and analyzing existing house decoration cases on the web, a new homeowner can find his or her decorative art patterns; however, the above decorative art pattern mining and discovery process is often time-consuming and boring due to the big volume of existing house decoration cases on the web. Therefore, it is becoming a challenging task to develop a time-efficient decorative art pattern mining and discovery method based on the available house decoration cases provided by historical users. Considering this challenge, a novel LSH-based similar homeowners clustering approach is proposed. A set of experiments are designed to validate the effectiveness and efficiency of the proposal.
\end{abstract}

\section{KEYWORDS}

Decorative Art, Group Decision-Making, Locality-Sensitive Hashing, Pattern Mining, Similar Users

\section{INTRODUCTION}

With the ever-increasing growth of real estate industry and the diversity of house owners' personalized preferences, people are apt to decorate their houses sufficiently to meet their respective aesthetic standards (Yuan, 2013; Barua, 2019; Yu, 2018; Chen, 2017; Beneðeld, 2019). For example, young house owners often prefer to decorate their houses according to modern decoration styles such as landscape painting style and cartoon style, while elder people may prefer great man style instead of other complicated ones. Today, house decoration has become a crucial and important industry all over the world and has provisioned people with various and diverse house decoration styles to meet the personalized requirements from different individuals.

With the gradual popularity of Web (Xue, 2020; Srinivasan, 2021; Kou, 2020; Sridharan, 2021; $\mathrm{Wu}, 2021$ ), people are apt to publish their house decoration cases on the Web for the purpose of case sharing and attraction of readers. On one hand, such shared house decoration cases offer new house owners more case references or decoration experiences, and on the other hand, place a heavy burden 
on the decoration style or art selection, because there are so many available house decoration cases generated from past decoration behaviors of historical users. For example, Fig.1 presents three kinds of decoration art works with distinct decoration styles (e.g., Space Formation with different elements, A Single Boat floating on a river, Night with static moon and stars).

Figure 1. Different decoration styles or arts

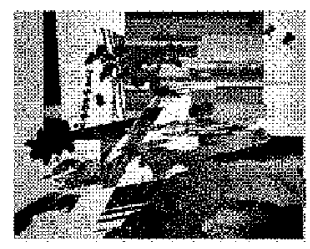

(a) Space Formation

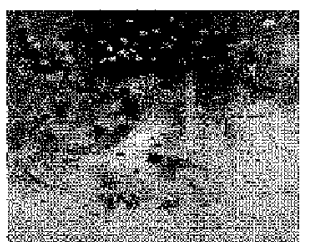

(b) A Single Bont

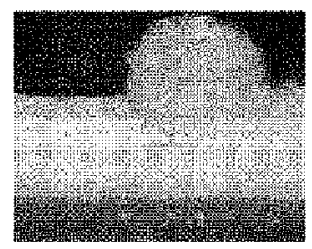

(c) Nightt

In this situation, a challenge is raised to help new house owners to find out their interested or preferred decoration arts or styles accurately and quickly. Recommender systems have been proven a promising way to realize the above goal. While existing recommendation solutions seldom optimize recommendation accuracy and privacy-preservation simultaneously. Inspired by this challenge, a novel decorative art pattern mining and discovery approach (abbreviated as DPMD) is put forward in this paper, which is mainly based on the Locality-Sensitive Hashing technique (LSH) (Qi, 2020; Qi et al, 2020) that is widely adopted and welcome in the domain of information retrieval. Generally, the major contributions in this research are two-fold:

(1) We introduce a novel LSH-based similar house owners clustering approach DPMD, to achieve time-efficient and privacy-aware decorative art work recommendations. This way, we can balance user privacy and recommendation accuracy well.

(2) We design a set of experiments to validate the effectiveness and efficiency of our proposal in terms of making decorative art pattern mining and discovery. Through comparisons with related approaches, we prove the feasibility of our proposed decorative art pattern mining and discovery solution for house owners.

The reminder of this paper is organized as follows. Section 2 presents a motivating example to illustrate the research value and significance of this paper. In Section 3, we elaborate the concrete steps of our proposed decorative art pattern mining and discovery solution based on the historical house decoration cases and ratings on various decoration elements. A group of experiments are designed and executed in Section 4, through which we demonstrate the effectiveness and efficiency of our proposed resolution. At last, in Section 5, we conclude this research work and point out the possible research topics in the future work.

\section{MOTIVATION}

As Fig.1 shows, each decorative art work is often comprised of a series of decorative elements such as image, color, texture and material. Two users (i.e., house owners), i.e., Tom and Judy rated some elements of decorative arts, whose rating values are shown in Fig.2 (e.g., $5^{*}$ and $4^{*}$; the larger, the better). In this situation, these rating values towards different decorative elements from historical users 
provide a promising basis to evaluate the decorative preferences of Tom and Judy. In this situation, to judge or evaluate whether Tom and Judy have the same decorative preferences towards houses, we need to calculate the similarity degree of Tom and Judy according to their ratings towards different elements.

Figure 2. User rating-based decorative pattern mining and discovery: an example

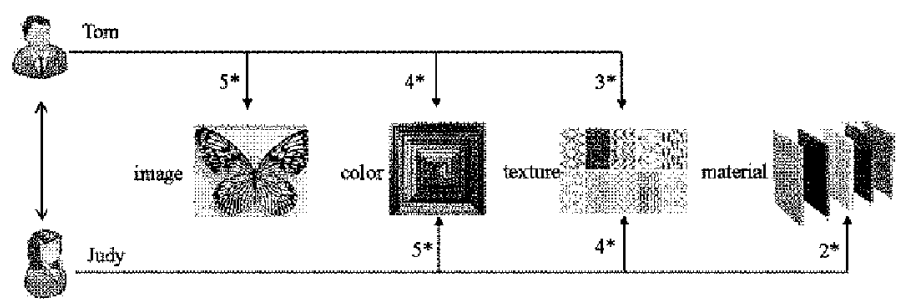

Traditional user preference similarity calculation methods such as collaborative filtering (CF) are often time-consuming as there are often too many user-element rating records (Wang, 2021; Liu, 2021). In addition, CF-based user preference similarity calculation methods need to read all the sensitive user rating records, which raises users' concerns on privacy leakage (Xu, 2020; Khazbak, 2020; Chen, 2019; Liu, 2019; Xu, 2020; Liu, 2017). Therefore, we need to address users' concerns from the above two perspectives. Inspired by this bservation, we bring forth a novel decorative art pattern mining approach based on LSH technique, i.e., DPMD. The details of the proposed DPMD approach are introduced in the next section.

\section{METHOD}

In general, the proposed decorative art pattern mining and discovery algorithm, i.e., DPMD consists of the following four steps. First, we extract representative features of decorative art works. Second, we transform the user ratings over the extracted features into a user vector based on LSH (the basic idea of LSH is that we can use an LSH function to convert a sensitive user vector into a less-sensitive user index; furthermore, if two users have the same index value, then they can be considered as similar with high probability), which often contains less user privacy and can denote the personalized preferences of the user. Third, we cluster users with similar rating preferences when selecting decorative art works, through which we can further discover the users with close preferences. Fourth, we recommend appropriate decorative art works to a target user based on his or her similar users. Fig.3 summarizes the above four steps with a more intuitive flowchart.

Step 1: Feature extraction of decorative art works.

For a decorative art work (e.g., the three works with diverse styles in Fig.1), there are often some attributes that can depict the overall style of the decorative art work, such as colors, textures, images, materials, brands, producers, and so on (see Fig.2). From the above attributes, we can extract the common focuses of users when they rate a decorative art work, e.g., (color, texture, image, material); thus, we can use the extracted features to represent the major characteristics of decorative art works. Step 2: Model user ratings on features into a preference vector.

For a decorative art work, a user often left a set of ratings over the decorative art work's features. For example, user Tom may rate a decorative art work he bought or selected with a score vector $\left(5^{*}\right.$, $3^{*}, 3^{*}, 4^{*}$ ) corresponding to the feature vector (color, texture, image, material). Thus, a user may leave multiple rating vectors towards his or her selected decorative art works. Here, we take their average rating vector. For example, if Tom selected 2 decorative art works and their rating vectors 


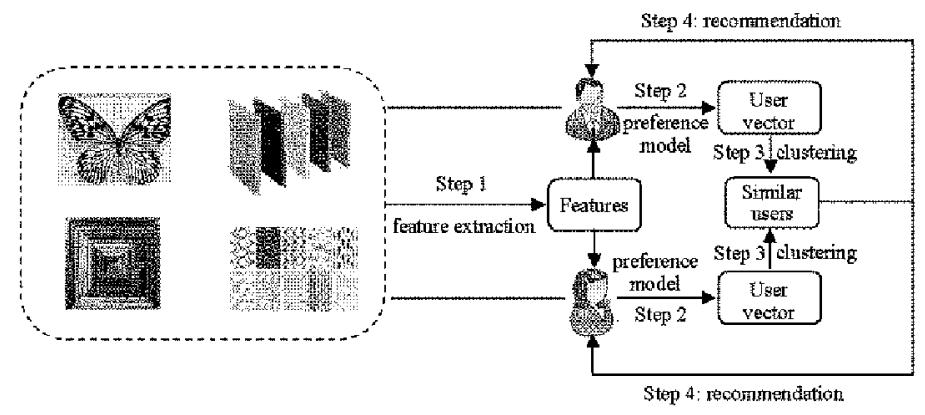

are $V_{\text {Tom }, 1}\left(5^{*}, 3^{*}, 3^{*}, 4^{*}\right)$ and $V_{\text {Tom }, 2}\left(3^{*}, 5^{*}, 1^{*}, 4^{*}\right)$, respectively; then the average rating vector for Tom would be $V_{\text {Tот }}\left(4^{*}, 4^{*}, 2^{*}, 4^{*}\right)$. In this situation, $V_{\text {Tот }}\left(4^{*}, 4^{*}, 2^{*}, 4^{*}\right)$ could be regarded as the rating preferences of Tom regarding the decorative art works.

Step 3: Cluster users into different groups based on their preference vectors.

After Step 1- Step 2, we can get an average rating preference vector for each user, e.g., $V_{\text {Tom }}\left(4^{*}\right.$, $\left.4^{*}, 2^{*}, 4^{*}\right), V_{J a c k}\left(3^{*}, 5^{*}, 4^{*}, 3^{*}\right), V_{J u d y}\left(5^{*}, 3^{*}, 2^{*}, 5^{*}\right)$. Next, we need to analyze these rating preference vectors and mine the potential decorative art patterns existing in historical user rating data. Such a preference analysis process is often time-consuming as there are so many user rating data shared in various house decoration websites or platforms. In addition, users' rating data are often sensitive enough as they contain partial privacy of users (Wang, 2020; Zhang, 2020; Xu et al, 2020; Sun, 2021; Qin Liu, 2019; Tianen Liu, 2020). Hence, from the security perspective (Alaoui, 2021; Xiaolong Xu, 2020; Guezzaz, 2021; Xiang Chen, 2020), it is becoming a challenge to develop a time-efficient and privacy-aware decorative art pattern mining and discovery method.

Considering the above challenges, LSH technique is employed to achieve the time-efficient and privacy-aware decorative art pattern mining and discovery purpose, as LSH is essentially an effective similar neighbor search method with privacy-preservation capability and a low time complexity $\mathrm{O}(1)$. The basic idea of LSH-based decorative art pattern mining and discovery solution is: we first convert the sensitive user rating vectors into less-sensitive index and then recognize those users with similar rating preferences based on index values with less sensitivity.

Next, we clarify the concrete index creation process for users. Here, we assume that there are $n$ features for decorative art works, denoted by $V\left(f e_{1}, f e_{2}, \ldots, f e_{n}\right)$, and there are $m$ users who left ratings, denoted by $U=\left\{u_{1}, u_{2}, \ldots, u_{m}\right\}$. As $f e_{1}, f e_{2}, \ldots$ and $f e_{n}$ are all numeric values, we generate the corresponding LSH functions as in (1)-(2). Here, $W$ denotes an $n$-dimensional vector and each dimensional value of $W$, i.e., $w_{j}$ is a random value among range $(-1,1)$.

$W=\left(w_{1}, w_{2}, \ldots, w_{n}\right)$

$w_{j}=\operatorname{random}(-1,1)(j=1,2, \ldots, n)$

Next, we get the dot product of vectors $V$ and $W$ and then obtain a real value $h$ as in (3). Here, we make the mapping or conversion in (4) for $h$ value. We repeat the calculation process formalized in (1)-(4) $p$ times, after which we get $p$ values of $h$, i.e., $h_{1}, h_{2}, \ldots, h_{p}$. Thus, we derive a Boolean value vector $H\left(h_{1}, h_{2}, \ldots, h_{p}\right)$. This vector can be considered as the index value of a user. Then for 


\begin{tabular}{|c|c|c|c|c|}
\hline & $W_{1}{ }_{1} H_{1}$ & $\mathrm{~W}_{2}{ }^{\circledR H_{2}}$ & $\ldots$ & $W_{m}{ }_{m}$ \\
\hline$Z_{1}$ & $W_{1} \circledast(0,1,1,0)$ & $W_{2}{ }^{\circledR}(0,0,1,1)$ & $\ldots$ & $W_{m}{ }^{\circledR}(0,0,0,1)$ \\
\hline$Z_{2}$ & $W_{1}{ }^{\circledR}(1,1,0,0)$ & $W_{2}{ }^{\circledR}(1,1,0,0)$ & $\ldots$ & $W_{m}{ }^{\circledR}(1,1,0,1)$ \\
\hline$\ldots$ & $\ldots$ & & $\ldots$ & $\ldots$ \\
\hline$Z_{q}$ & $W_{1}{ }^{\circledR}(1,0,1,1)$ & $W_{2}{ }^{\circledR}(0,1,1,1)$ & $\ldots$ & $W_{m}{ }^{\circledR}(1,0,1,0)$ \\
\hline
\end{tabular}

each user in set $U$, we can create an index and afterwards, we get the index values for all the $m$ users in set $U$, i.e., $H_{1}, H_{2}, \ldots, H_{m}$.

This way, the rating vector $W$ of each user is converted into a corresponding index $H$, whose advantages are two-fold: (1) the mapping or hashing process described in (1)-(4) is often done offline, whose time complexity is $\mathrm{O}(1)$; such a low time complexity can enable a quick index creation without wasting much computational time. (2) with index values $H_{1}, H_{2}, \ldots, H_{m}$, we can make the subsequent similar user clustering process without involving the sensitive user rating vectors $W_{1}, W_{2}$, $\ldots, W_{m}$, which enables the decorative art pattern mining and discovery process a good capability of privacy-preservation.

$h=V * W=\left(f e_{1}, f e_{2}, \ldots, f e_{n}\right) *\left(w_{1}, w_{2}, \ldots, w_{n}\right)^{T}=\sum_{j=1}^{n} f e_{j} * w_{j}$

$h= \begin{cases}1 & \text { if } h>0 \\ 0 & \text { if } h \leq 0\end{cases}$

Thus, the mappings from $W$ to $H$ forms an LSH table (denoted by $Z$ ), which is exampled in Table 1. Table 1 presents the examples of $q$ LSH tables: $Z_{1}, Z_{2}, \ldots, Z_{q}$. Then according to the rules of $\mathrm{LSH}$, two users $u_{i}$ and $u_{j}$ (their corresponding rating preference vectors are $W_{i}$ and $W_{j}$, respectively) are similar if and only if equation in (5) is satisfied. For example, users $u_{1}$ and $u_{2}$ can be regarded as similar because $H_{1}=H_{2}$ holds in table $Z_{2}$ (in $Z_{2}$, the index values for $u_{1}$ and $u_{2}$ are both $(1,1,0,0)$ ). This way, for a target user $u^{\#}$, we can get his or her similar users (denoted by $\operatorname{Sim}\left(u^{\#}\right)$ ) according to the created $p$ tables. In other words, $\operatorname{Sim}\left(u^{\#}\right)$ can be calculated by (6).

$u_{i}$ and $u_{j}$ are similar iff $H_{i}=H_{j}$ in any of $Z_{1}, Z_{2}, \ldots, Z_{q}$

$\operatorname{Sim}\left(u^{\#}\right)=\left\{u_{i} \mid u_{i} \hat{\mathrm{I}} U, u_{i}\right.$ and $u^{\#}$ are similar $\}$

Step 4: Decorative art work recommendations for a target user.

In Step 3, we have obtained a set of similar users of the target user $u^{\#}$, i.e., $\operatorname{Sim}\left(u^{\#}\right)$. Next, with the known ratings of users in set $\operatorname{Sim}\left(u^{\#}\right)$, we can predict the missing rating data of $u^{\#}$ over certain decorative elements in set $V\left(f e_{1}, f e_{2}, \ldots, f e_{n}\right)$. The basis rationale of rating prediction is that similar users are probably owning the same ratings over the identical decorative elements. In concrete, the rating prediction process can be formalized by (7). Here, $u_{i} f e_{j}$ denotes user $u_{i}$ 's rating value over feature $f e_{j}(i=1,2, \ldots, m ; j=1,2, \ldots, n) ;\left|\operatorname{Sim}\left(u^{\#}\right)\right|$ represents the size of vector $\operatorname{Sim}\left(u^{\#}\right)$. In other 
words, $u^{\#} . f e_{j}$ is equal to the average rating of the similar users of $u^{\#}$ over feature $f e_{j}$. Finally, with the predicted $u^{\#} . f e_{j}$ value, we can make appropriate decorative art work recommendations.

$u^{\#} \cdot f e_{j}=\frac{1}{\left|\operatorname{Sim}\left(u^{\#}\right)\right|} * \sum_{u_{i} \in \operatorname{Sim}\left(u^{*}\right)} u_{i} \cdot f e_{j}$

\section{EXPERIMENTS}

A set of experiments are deployed to validate the feasibility of our proposed DPMD method. Experimental dataset is WS-DREAM (Qi, 2020). The dataset records the feedback data of 339 users towards 5825 items. The two methods for comparisons include user-based CF (User-CF) (Qi et al, 2020) and item-based CF (Item-CF) (Qi et al, 2020). Evaluation metrics are MAE (Qi et al, 2020) (for measuring system accuracy, smaller is better) and Time cost (Qi et al, 2020) (for measuring system efficiency, smaller is better). The experimental environments include Python 3.6 programming language and Lenovo computer (2.6GHz processor, 8.0GB RAM, Windows 10 operating system). Each experiment is run 100 times and finally their average results are used for comparison illustrations. Here, $m$ and $n$ denote the sizes of user set and feature set, respectively.

In concrete, four sets of experimental comparisons are enacted and displayed, respectively.

\section{Profile 1: Accuracy of DPMD, User-CF and Item-CF.}

In this profile, we measure the accuracy of DPMD and compare it with two ones: User-CF and Item-CF. Concrete running results are reported in Fig.4. Parameters $p$ (size of hash function set) and $q$ (size of hash table set) are both equal to 10. In Fig.4(a), $m$ is varied from 50 to 300 and $n$ is equal to 5000; while in Fig.4(b), $n$ is varied from 1000 to 5000 and $m$ is equal to 300. It can be seen from Fig. 4 that the MAE value of DPMD algorithm is always smaller than those of User-CF and Item$\mathrm{CF}$, which indicates a higher accuracy performance of DPMD. It can be explained by the following fact: DPMD algorithm utilizes LSH technique to search for similar users of a target user, which can guarantee that only those similar users are returned for subsequent missing rating data prediction. As a result, the prediction accuracy is increased accordingly.

\section{Profile 2: Efficiency of DPMD, User-CF and Item-CF.}

In this profile, we measure the execution efficiency of the proposed DPMD method and compare it with the other two ones: User-CF and Item-CF. Concrete running results are reported in Fig.5. Here, $p=q=10$. In Fig.5(a), $m$ is varied from 50 to 300 and $n=5000$; while in Fig.5(b), $n$ is varied from 1000 to 5000 and $m=300$. It can be seen from Fig.5 that the computational time of DPMD is superior to the other two competitive approaches as the LSH-based similar user clustering solution is rather time-efficient (complexity is approaching $\mathrm{O}(1)$ ). The low complexity also shows the applicability of our proposal in deal with big rating data. 
Figure 4. Accuracy comparisons of three algorithms

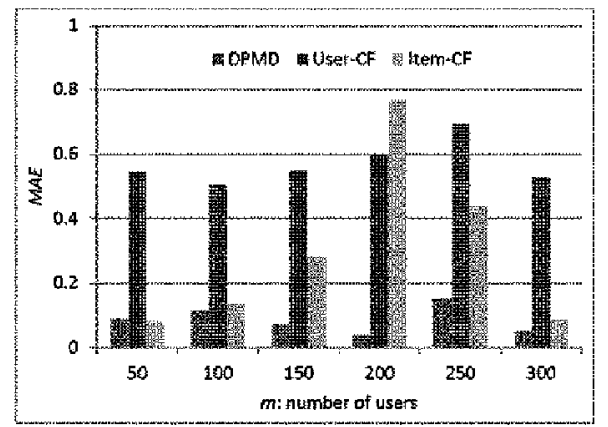

(a) $n=5000$

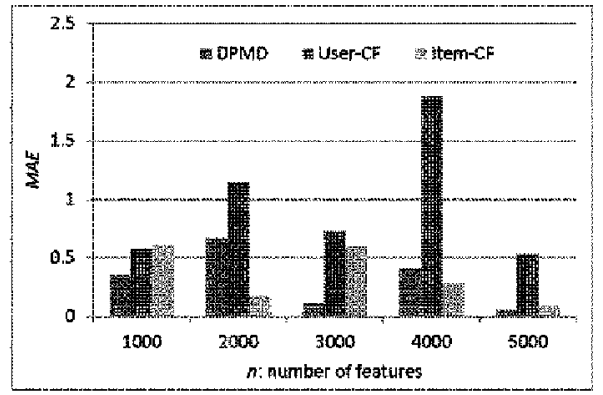

(b) $m=300$

Figure 5. Execution efficiency comparisons of three algorithms

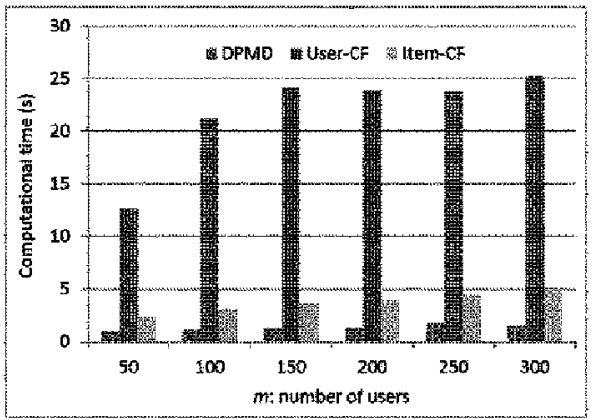

(a) $n=5000$

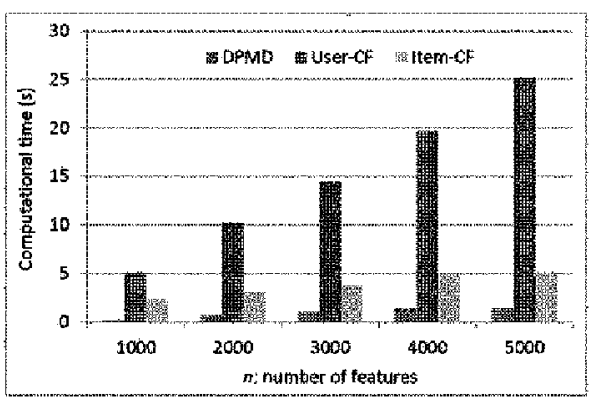

(b) $m=300$ 


\section{PROFILE 3: ACCURACY OF DPMD WITH RESPECT TO $P$ AND $Q$.}

According to Section 4, two key factors are involved in DPMD method, i.e., $p$ (size of hash function set) and $q$ (size of hash table set). Considering their influences, we design a test to measure the correlation between MAE of DPMD and factors $p$ and $q$. Experimental results are presented in Fig.6. The reported data in Fig. 6 shows a decline of MAE (i.e., an increment of prediction accuracy) with the growth of factor $p$. The results can be analyzed as follows: a larger $p$ often means a stricter evaluation condition when we select a qualified similar user of a target user; as a result, only the "most similar" friends of the target user could be found for subsequent missing rating data prediction. Therefore, the prediction accuracy would be raised accordingly.

Figure 6. Accuracy of DPMD w.r.t. $(p, q)$

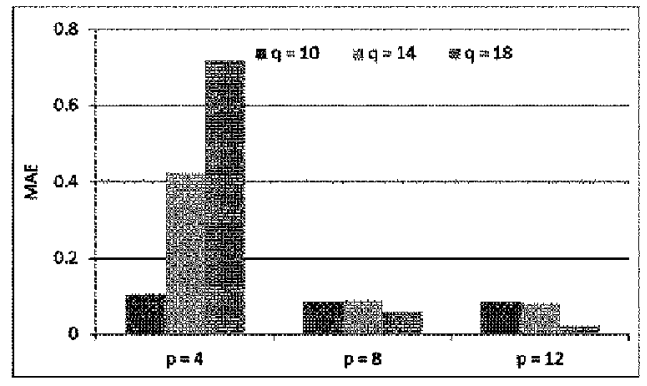

\section{Profile 4: Efficiency of DPMD with respect to $p$ and $q$.}

As discussed in Profile 3, $p$ and $q$ are two key factors that can influence the prediction performances of the proposed DPMD algorithm. Inspired by this observation, we design an experiment to observe the relationship between the computational time of DPMD and the parameter pair (p, q), whose results are demonstrated in Fig.7. As the figure shows, the consumed time of DPMD algorithm decreases with the growth of $p$, which could be explained as follows: a larger $p$ often means a stricter filtering condition when we evaluate a qualified similar user of a target user; as a result, only a smaller

Figure 7. Execution efficiency of DPMD w.r.t. $(p, q)$

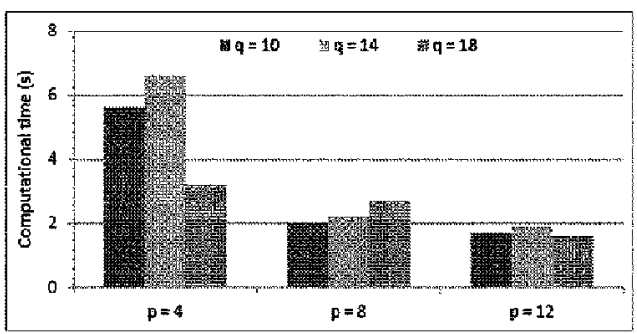

number of "most similar" friends of the target user could be found for subsequent missing rating data prediction. Therefore, less time cost is needed to finish the final missing rating prediction process. 


\section{CONCLUSION AND FUTURE WORK}

With the ever-increasing personality of user preferences towards decorative arts or styles, house owners are currently confronted with more and more decorative alternatives or candidates, which plays a heavy burden on the final decorative style selection decision-makings of house owners. In this situation, it is becoming a valuable and significant research task to mine and discover the same decorative patterns and then recommend appropriate decorative art works to a target user based on the users with the same decorative pattern. However, traditional solutions are often time-consuming and sensitive. Considering this challenge, a novel decorative art pattern mining and discovery method named DPMD is introduced in this paper, to achieve time-efficient and privacy-aware decorative art work recommendations. Finally, we validate the feasibility of DPMD via a set of experiments.

In the future research, we would like to further investigate more influencing factors in users' decoration selection decision-makings, such as time and location information of users (Malek, 2021; Xiang Chen, 2021). In addition, more performance metrics (e.g., network load (Xiaolong Xu et al, 2020; Li, 2021; Xiaolong Xu, Bowen Shen, 2020; Yun Li, 2021; Bi, 2021; Yun Li et al, 2020), diversity (Jianxin Li, 2020; Cai, 2020)) should be taken into considerations besides accuracy and time cost. Third, how to make full use of the cutting-edge learning techniques (e.g., deep learning, machine learning, transfer learning) (Huang, 2020; Xue, 2019; Yuvaraj, 2021) to further improve the algorithm performances is still requiring challenging efforts. Finally, our proposed DPMD method still needs to be improved by measuring and quantifying its capability of privacy-preservation; therefore, we will further investigate current privacy protection solutions such as (Zheng, 2020; Kaiyang Li, 2020; Lina Wang, 2020; Zhipeng Cai, 2020; Zheng, 2017) to pursue a feasible and scientific privacy measurement solution. 


\section{REFERENCES}

Beneðeld, J. D., \& Sirmans, S. (2019). Observable Agent Effort and Limits to Innovation in Residential Real Estate. Journal of Real Estate Research, 41(1), 1-36.

Bi, R., Liu, Q., Ren, J., \& Tan, G. (2021). Utility Aware Offloading for Mobile-Edge Computing. Tsinghua Science and Technology, 26(2), 239-250.

Cai, T., Li, J., \& Ajmal, S. (2020). Target-aware Holistic Influence Maximization in Spatial Social Networks. IEEE Transactions on Knowledge and Data Engineering. Advance online publication. doi:10.1109/TKDE.2020.3003047

Cai, Z., \& Zheng, X. (2020). A Private and Efficient Mechanism for Data Uploading in Smart Cyber-Physical Systems. IEEE Transactions on Network Science and Engineering, 7(2), 766-775.

Chen, M., Gao, B., \& Zhu, Z. (2017). Analysis on Regional Difference of Development of Elderly Care Real Estate in Gannan Area. Agricultural Science and Technology, 18(10), 1979-1988.

Chen, X., Mu, Y., Liu, K., Cui, Z., \& Ni, C. (2021). Revisiting Heterogeneous Defect Prediction Methods: How Far Are We? Information and Software Technology, 130, 106441.

Chen, X., Mu, Y., Qu, Y., Ni, C., Liu, M., He, T., \& Liu, S. (2020). Do Different Cross-project Defect Prediction Methods Identify the Same Defective Modules? Journal of Software: Evolution and Process, 32(5), e2234.

Chen, X., Zhao, Y., Cui, Z., Meng, G., Liu, Y., \& Wang, Z. (2019). Large-scale Empirical Studies on EffortAware Security Vulnerability Prediction Methods. IEEE Transactions on Reliability, 69(1), 70-87.

El Arbi, A. A., Stéphane, C. K. T., Hartini, S., Rustam, Z., Silkan, H., \& Agoujil, S. (2021). Improvement in Automated Diagnosis of Soft Tissues Tumors Using Machine Learning. Big Data Mining and Analytics, 4(1), $33-46$.

Guezzaz, A., Asimi, Y., Azrour, M., \& Asimi, A. (2021). Mathematical Validation of Proposed Machine Learning Classifier for Heterogeneous Traffic and Anomaly Detection. Big Data Mining and Analytics, 4(1), 18-24.

Huang, H., Lin, J., Wu, L., Fang, B., Wen, Z., \& Sun, F. (2020). Machine Learning-Based Multi-Modal Information Perception for Soft Robotic Hands. Tsinghua Science and Technology, 25(02), 255-269.

Khazbak, Y., Fan, J., Zhu, S., \& Cao, G. (2020). Preserving Personalized Location Privacy in Ride-Hailing Service. Tsinghua Science and Technology, 25(6), 743-757.

Kou, H., Liu, H., Duan, Y., Gong, W., Xu, Y., Xu, X., \& Qi, L. (2020). Building Trust/Distrust Relationships on Signed Social Network through Privacy-aware Link Prediction. Applied Soft Computing. Advance online publication. doi:10.1016/j.asoc.2020.106942

Li, J., Cai, T., Deng, K., Wang, X., Sellis, T., \& Xia, F. (2020). Community-diversified Influence Maximization in Social Networks. Information Systems, 92, 1-12.

Li, K., Lu, G., Luo, G., \& Cai, Z. (2020). Seed-free Graph De-anonymiztiation with Adversarial Learning. The 29 th ACM International Conference on Information and Knowledge Management.

Li, Y., Ma, H., Wang, L., Mao, S., \& Wang, G. (2020). Optimized Content Caching and User Association for Edge Computing in Densely Deployed Heterogeneous Networks. IEEE Transactions on Mobile Computing. Advance online publication. doi:10.1109/TMC.2020.3033563

Li, Y., Xia, S., Yang, Q., Wang, G., \& Zhang, W. (2021). Lifetime-Priority-Driven Resource Allocation for WNV-Based Internet of Things. IEEE Internet of Things Journal, 8(6), 4514-4525.

Li, Y., Zhang, Z., Xia, S., \& Chen, H.-H. (2021). A Load-Balanced Re-embedding Scheme for Wireless Network Virtualization. IEEE Transactions on Vehicular Technology. Advance online publication. doi:10.1109/ TVT.2021.3064257

Liu, Q., Hou, P., Wang, G., Peng, T., \& Zhang, S. (2019). Intelligent Route Planning on Large Road Networks with Efficiency and Privacy. Journal of Parallel and Distributed Computing, 133, 93-106. 
Liu, Q., Tian, Y., Wu, J., Peng, T., \& Wang, G. (2019). Enabling Verifiable and Dynamic Ranked Search Over Outsourced Data. IEEE Transactions on Services Computing. Advance online publication. doi:10.1109/ TSC.2019.2922177

Liu, Q., Wang, G., Li, F., Yang, S., \& Wu, J. (2017). Preserving Privacy with Probabilistic Indistinguishability in Weighted Social Networks. IEEE Transactions on Parallel and Distributed Systems, 28(5), 1417-1429.

Liu, T., Wang, Y., Li, Y., Tong, X., Qi, L., \& Jiang, N. (2020). Privacy Protection based on Stream Cipher for Spatio-temporal Data in IoT. IEEE Internet of Things Journal, 7(9), 7928-7940.

Liu, Y., Wang, F., Yang, Y., Zhang, X., Wang, H., Dai, H., \& Qi, L. (2021). An Attention-based Category-aware GRU Model for Next POI Recommendation. International Journal of Intelligent Systems. Advance online publication. doi:10.1002/int.22412

Malek, Y. N., Najib, M., Bakhouya, M., \& Essaaidi, M. (2021). Multivariate Deep Learning Approach for Electric Vehicle Speed Forecasting. Big Data Mining and Analytics, 4(1), 56-64.

Qi, L., Hu, C., Zhang, X., Khosravi, M. R., Sharma, S., Pang, S., \& Wang, T. (2020). Privacy-aware Data Fusion and Prediction with Spatial-Temporal Context for Smart City Industrial Environment. IEEE Transactions on Industrial Informatics. Advance online publication. doi:10.1109/TII.2020.3012157

Qi, L., Wang, X., Xu, X., Dou, W., \& Li, S. (2020). Privacy-Aware Cross-Platform Service Recommendation based on Enhanced Locality-Sensitive Hashing. IEEE Transactions on Network Science and Engineering. Advance online publication. doi:10.1109/TNSE.2020.2969489

Sridharan, R., \& Domnic, S. (2021). Placement for Intercommunicating Virtual Machines in Autoscaling Cloud Infrastructure: Autoscaling and Intercommunication Aware Task Placement. Journal of Organizational and End User Computing. Advance online publication. doi:10.4018/JOEUC.20210301.oa2

Srinivasan, S., \& Dhinesh, B. L. D. (2021). A Bio-Inspired Defensive Rumor Confinement Strategy in Online Social Networks. Journal of Organizational and End User Computing. Advance online publication. doi:10.4018/ JOEUC.2021010103

Sun, Z., Wang, Y., Cai, Z., Liu, T., Tong, X., \& Jiang, N. (2021). A Two-stage Privacy Protection Mechanism Based on Blockchain in Mobile Crowdsourcing. International Journal of Intelligent Systems. Advance online publication. doi:10.1002/int.22371

Uchchash Barua, M. (2019). Real Estate Recommendation Using Historical Data and Surrounding Environments. International Journal of Information Engineering \& Electronic Business, 11(5), 33-39.

Wang, F., Zhu, H., Srivastava, G., Li, S., Khosravi, M. R., \& Qi, L. (2021). Robust Collaborative Filtering Recommendation with User-Item-Trust Records. IEEE Transactions on Computational Social Systems. doi:10.1109/TCSS.2021.3064213

Wang, L., Zhang, X., Wang, R., Yan, C., Kou, H., \& Qi, L. (2020). Diversified Service Recommendation with High Accuracy and Efficiency. Knowledge-Based Systems, 204, 106196. Advance online publication. doi:10.1016/j.knosys.2020.106196

Wang, Y., Gao, Y., Li, Y., \& Tong, X. (2020). A Worker-Selection Incentive Mechanism for Optimizing PlatformCentric Mobile Crowdsourcing Systems. Computer Networks, 107, 107144.

Wu, W., Ma, S., Su, Y., \& Wu, C.-H. (2021). Double-Layer Learning, Leaders' Forgetting, and Knowledge Performance in Online Work Community Organizations. Journal of Organizational and End User Computing. Advance online publication. doi:10.4018/JOEUC.2021010105

Xu, X., Huang, Q., Zhu, H., Sharma, S., Zhang, X., Qi, L., \& Md, Z. A. B. (2020). Secure Service Offloading for Internet of Vehicles in SDN-Enabled Mobile Edge Computing. IEEE Transactions on Intelligent Transportation Systems. Advance online publication. doi:10.1109/TITS.2020.3034197

Xu, X., Li, H., Xu, W., Liu, Z., Yao, L., \& Dai, F. (2020). Artificial Intelligence for Edge Service Optimization in Internet of Vehicles: A Survey. Tsinghua Science and Technology. Advance online publication. doi:10.26599/ TST.2020.901 
Xu, X., Mo, R., Yin, X., Khosravi, M. R., Aghaei, F., Chang, V., \& Li, G. (2020). PDM: Privacy-Aware Deployment of Machine-Learning Applications for Industrial Cyber-Physical Cloud Systems. IEEE Transactions on Industrial Informatics. Advance online publication. doi:10.1109/TII.2020.3031440

Xu, X., Shen, B., Ding, S., Srivstava, G., Bilal, M., Khosravi, M. R., \& Varun, G. (2020). Menon, Mian Ahmed Jan, Maoli Wang, Service Offloading with Deep Q-Network for Digital Twinning Empowered Internet of Vehicles in Edge Computing. IEEE Transactions on Industrial Informatics. Advance online publication. doi:10.1109/ TII. 2020.3040180

Xu, Y., Zhang, C., Wang, G., Qin, Z., \& Zeng, Q. (2020). A Blockchain-enabled Deduplicatable Data Auditing Mechanism for Network Storage Services. IEEE Transactions on Emerging Topics in Computing. 10.1109/ TETC.2020.3005610

Xu, Y., Zhang, C., Zeng, Q., Wang, G., Ren, J., \& Zhang, Y. (2020). Blockchain-Enabled Accountability Mechanism Against Information Leakage in Vertical Industry Services. IEEE Transactions on Network Science and Engineering. 10.1109/TNSE.2020.2976697

Xue, X., Chen, Z., Wang, S., Feng, Z., Duan, Y., \& Zhou, Z. (2020). Value Entropy: A Systematic Evaluation Model of Service Ecosystem Evolution. IEEE Transactions on Services Computing. Advance online publication. doi:10.1109/TSC.2020.3016660

Xue, X., \& Wang, S. (2019). Social Learning Evolution (SLE): Computational Experiment-based Modeling Framework of Social Manufacturing. IEEE Transactions on Industrial Informatics, 15(6), 3343-3355.

Yu, Y., Wang, C., Zhang, L., Gao, R., \& Wang, H. (2018). Geographical Proximity Boosted Recommendation Algorithms for Real Estate. In International Conference on Web Information Systems Engineering. Springer.

Yuan, X., Lee, J.-H., Kim, S.-J., Kim, Y.-H., \& Toward, A. (2013). User-Oriented Recommendation System for Real Estate Websites. Information Systems, 38(2), 231-243.

Yuvaraj, N., Srihari, K., Chandragandhi, S., Raja, R. A., Dhiman, G., \& Kaur, A. (2021). Analysis of ProteinLigand Interactions of SARS-CoV-2 Against Selective Drug Using Deep Neural Networks. Big Data Mining and Analytics, 4(2), 76-83.

Zhang, Y., Pan, J., Qi, L., \& He, Q. (2020). Privacy-Preserving Quality Prediction for Edge-based IoT Services. Future Generation Computer Systems. Advance online publication. doi:10.1016/j.future.2020.08.014

Zheng, X., \& Cai, Z. (2020). Privacy-Preserved Data Sharing towards Multiple Parties in Industrial IoTs. IEEE Journal on Selected Areas in Communications, 38(5), 968-979.

Zheng, X., Cai, Z., Li, J., \& Gao, H. (2017). Location-Privacy-Aware Review Publication Mechanism for Local Business Service Systems. The 36th Annual IEEE International Conference on Computer Communications (INFOCOM 2017).

Rongju Yao was born in 1982 in Linqu, China. From 2003 to 2007, studied at Jilin University of the Arts. From 2012 to December 2013, studied at Qingdao University and got master's degree. From February 2021, as a doctoral candidate at Yongheng University in the Philippines, majoring in animation, game and post-production. During this period, accumulated rich teaching experience and published many articles, patents and projects in journals.

Ashish Kr Luhach received Ph.D degree in department of computer science from Banasthali University, India. Dr. Luhach is working as Senior lecturer at The Papua New Guinea University of Technology, Papua New Guinea. He has more than a decade of teaching and research experience. Dr. Luhach also worked with various reputed universities and also holds administrate experience as well. Dr. Luhach has published more 80 research paper in reputed journals and conferences, which are indexed in various international databases. He is also edited various special issues in reputed journals, and he is Editor/Conference Co-chair for various conferences. Dr. Luhach is also editorial board members of various reputed journals. He is member of IEEE, CSI, ACM and IACSIT. 